\title{
Autonomous use of electrical energy by an artificial molecular machine
}

Giulio Ragazzon, ${ }^{\mathrm{a}, *}$, Marco Malferrari, ${ }^{\mathrm{b}}$ Arturo Arduini, ${ }^{\mathrm{c}}$ Andrea Secchi, ${ }^{\mathrm{c}}$ Stefania Rapino, ${ }^{\mathrm{b}, *}$ Serena Silvi, ${ }^{\text {b,d,* }}$ and Alberto Credi ${ }^{\mathrm{d}, \mathrm{e}}$

a Institut de Science et d'Ingégnierie Supramoléculaires (ISIS), Université de Strasbourg, CNRS, 8 allée Gaspard Monge, 67000 Strasbourg, France

${ }^{b}$ Dipartimento di Chimica "G. Ciamician", Università di Bologna, via Selmi 2, 40126 Bologna, Italy

c Dipartimento di Scienze Chimiche, della Vita e della Sostenibilità Ambientale, Parco Area delle Scienze 17/A, 43124 Parma, Italy

${ }^{d}$ CLAN-Center for Light-Activated Nanostructures (CLAN), Università di Bologna and Consiglio Nazionale delle Ricerche, via Gobetti 101, 40129 Bologna, Italy

e Dipartimento di Chimica Industriale "Toso Montanari", viale del Risorgimento 4, 40136 Bologna, Italy

The ability to exploit energy autonomously is one of the hallmarks of Life. ${ }^{1}$ Mastering such processes in artificial nanosystems can open unforeseen technological opportunities. ${ }^{2}$ In the last decades, light- and chemically-driven autonomous systems have been developed in relation to conformational motion and self-assembly. ${ }^{3,4,5,6}$ On the contrary, the autonomous exploitation of electrical energy remains essentially unexplored, despite being an attractive energy source. ${ }^{7,8}$ Herein we demonstrate the autonomous operation of an electrochemically-powered selfassembling nanomachine. Threading and dethreading motions of a pseudorotaxane take place autonomously in solution, between the electrodes of a scanning electrochemical microscope. ${ }^{9,10}$ This innovative actuation mode allows operating a molecular machine with an energy efficiency of $9 \%$, unprecedented in autonomous systems. The strategy is general and can be applied to any redox-driven system, including molecular pumps that perform work repetitively. ${ }^{11,12}$ Ultimately, our study brings molecular nanoscience one step closer to everyday technology.

Autonomous processes are ubiquitous in Nature and constitute one of the key aspects of Life. For this reason, scientists have attempted to create artificial systems that display autonomous behavior, i.e., operate under constant environmental conditions, harnessing energy from the surrounding environment. ${ }^{13,14,15,16,17,18}$ The minimal form of autonomous chemical system is composed by a cyclic sequence of reactions that occurs spontaneously. In such a non-equilibrium system, energy needs to be supplied to impart a directionality in the reaction network, leading to the emergence of a chemical current (Figure 1a). ${ }^{17,19,20,21}$ This directional flux becomes extremely important when it is associated with molecular motion, as it occurs in natural and artificial molecular machines. ${ }^{22,23}$

Biomolecular machines, such as ATP synthase, myosin, and kinesin, work autonomously by consuming chemical fuels. In fact, these devices ultimately catalyze the decomposition of their fuel while undergoing structural transformations. Inspired by natural systems, chemists have developed 
autonomous molecular machines such as light- $-3,24,25$ and chemically-driven ${ }^{4}$ rotary motors, switches, ${ }^{26,27,28,29,30}$ pumps, $^{5,6}$ walkers, $^{31}$ and actuators. ${ }^{32,33,34}$

a

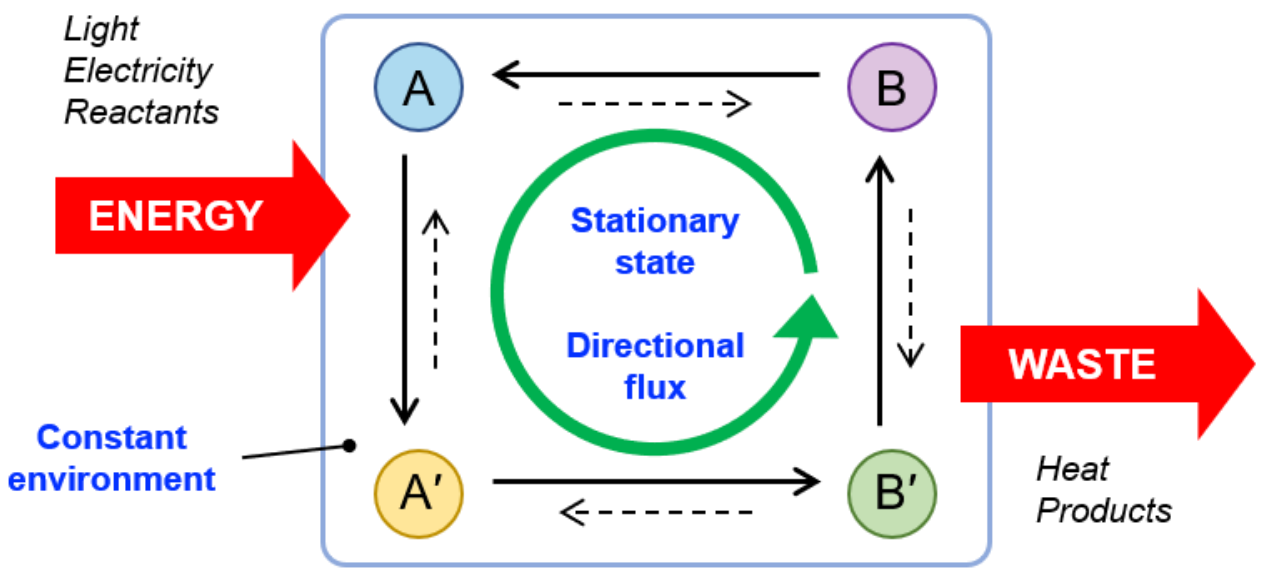

b

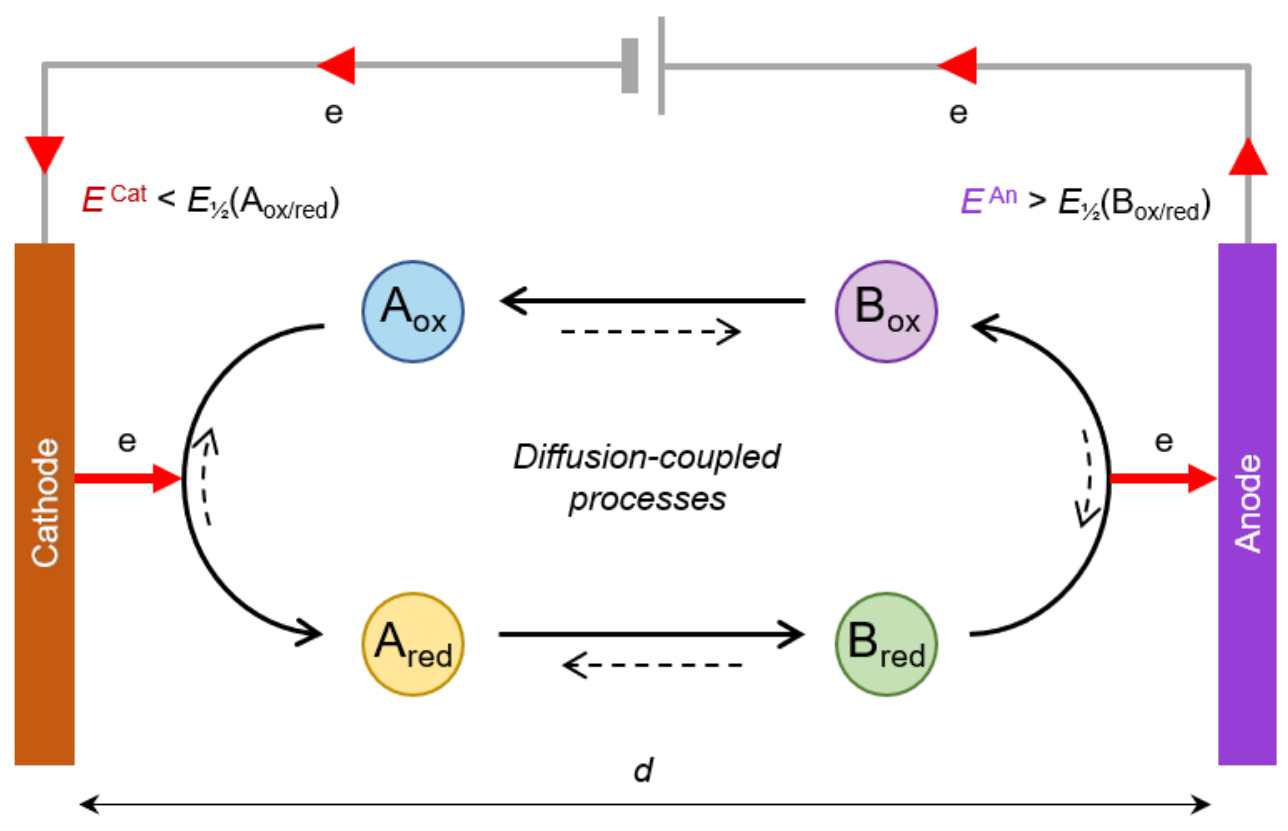

Figure 1. Autonomous chemical cycling sustained by an energy source. (a) The concept of autonomous operation of a system described as a cycle of chemical reactions. By exploiting an energy source, the chemical reaction cycle proceeds with a preferential direction, originating a mass flux. The system, under constant environmental conditions, reaches a non-equilibrium stationary state that is maintained until energy is supplied. In the case of a molecular machine, at least some of the transformations between $A, A^{\prime}, B$, and $B^{\prime}$ are molecular movements. Low-energy products and/or heat are also generated as a result of energy dissipation. (b) General scheme of the electrochemically driven sequence of reactions that lead to autonomous cycling between states $A$ and $B$, obtained by setting two appropriately spaced $(d)$ electrodes at different constant potential values $\left(E^{\mathrm{Cat}}, E^{\mathrm{An}}\right)$. The current flow from the cathode to the anode implies reduction/oxidation and diffusion of the components that are continuously formed in the switching cycle. 
Despite these achievements, the vast majority of artificial molecular machines reported so far cannot operate autonomously, and making devices of this kind remains a formidable research challenge. ${ }^{35,36,37}$ In particular, autonomous electrically-driven molecular machines are significantly underdeveloped in comparison with light- and chemically-driven ones. This fact is striking, considering the relevance of electricity in our society and the ease of producing electrical energy from renewable sources. The sole examples available to date are surface-mounted single-molecule rotors, whose operation relies on scanning tunneling microscopy (STM) experiments performed under special conditions (ultrahigh vacuum and very low temperature) that hamper real world applications. ${ }^{7,8}$ In these experiments, the molecule is part of an electric circuit in which the STM tip and the substrate are kept at different potentials.

The ability of redox-active molecular machines as electrically powered nanodevices, capable of exploiting electrical energy on the macroscopic scale under ambient conditions, has yet to be unfolded. For this purpose, general strategies that unlock the collective autonomous operation of electrochemically driven machines need to be found. This goal is particularly relevant for selfassembling systems, whose operation at the single-molecule level has significant fundamental and technical limitations.

To address the outlined challenge, one possibility is to rely on redox-active oscillating systems, where the origin of the emergent phenomena is rooted in the kinetic control of redox reactions. Albeit prominent examples have been reported, 38,39,40,41 this approach suffers from limited flexibility and it is difficult to generalize since it critically relies on specific kinetic features of the oscillating system that are difficult to tailor. An alternative, more viable option consists in interfacing the molecular machine with two electrodes operating at different potentials (Figure 1b). The use of a bipotentiostat enables the independent control of two electrodes, offering a general expedient to operate electrochemically-driven non-equilibrium systems. This concept was pioneered by Huskens and coworkers, who exploited the simultaneous but spatially distinct oxidation and reduction of a redox-active guest to control its binding to a monolayer of hosts, resulting in the formation of chemical gradients on a surface. ${ }^{42}$

Herein, we demonstrate the autonomous operation of a redox-driven molecular machine fuelled by electrical energy. We envisioned that this goal could be demonstrated by means of two electrodes, using a scanning electrochemical microscope (SECM) in a bipotentiostatic configuration. Such a setup enables a careful, simultaneous, and independent control of the electrode potentials and their relative distance (Figure $1 \mathrm{~b}$ ). The use of an ultramicroelectrode (UME), and the capability to position it with submicrometric precision with respect to a second electrode surface, enable the investigation of the oxidation-reduction processes occurring at both the UME and the biased substrate. ${ }^{43,44}$ These features, crucial to the present investigation, have been employed to study several molecular systems and materials. ${ }^{9,10}$ Yet, to the best of our knowledge, they were never exploited to operate molecular machines.

To test our hypothesis, we focused on an electroactive pseudorotaxane, $[\mathbf{1} \supset \mathbf{2}]^{2+}$, composed by tris(N-phenylureido)calix[6]arene $\mathbf{1}$ and 1,10-dipentyl-4,4'-bipyridinium $\mathbf{2}^{2+}$ (Figure 2a). Indeed, $\mathbf{1}$ is a well-known host for the latter compound and analogous axles; the pseudorotaxane formed with $2^{2+}$ has a stability constant $K_{\mathrm{ox}}=5.0 \times 10^{6} \mathrm{M}^{-1}$ in $\mathrm{CH}_{2} \mathrm{Cl}_{2} \cdot{ }^{45}$ The assembly and disassembly of these components can be controlled electrochemically, according to the square scheme reported in Figure $2 b .{ }^{46}$ Specifically, the reduction of $[\mathbf{1} \supset \mathbf{2}]^{2+}$ to $[\mathbf{1} \supset \mathbf{2}]^{+\bullet}$ results in the complete dethreading of $\mathbf{2}^{+\bullet}$. This process can be induced and monitored in cyclic voltammetry (CV) experiments (Figure 2c), where a reduction current is observed for potential values more negative than ca. $-0.7 \mathrm{~V}$ vs SCE, indicative of the reduction of $[\mathbf{1} \supset \mathbf{2}]^{2+}$. In the return scan, the observation of an oxidation current at ca. $-0.3 \mathrm{~V}$ 
- a potential typical of the oxidation of free $\mathbf{2}^{+\bullet}$ to $\mathbf{2}^{2+}-$ highlights the fast dethreading of $\mathbf{2}^{+\bullet}$ from $\mathbf{1}$ on the CV time scale.

At potential values more positive than $-0.3 \mathrm{~V}$ both $\mathbf{2}^{2+}$ and $[\mathbf{1} \supset \mathbf{2}]^{2+}$ are thermodynamically stable, whereas at values more negative than $-0.7 \mathrm{~V}$ the reduced forms $\mathbf{2}^{+\bullet}$ and $[\mathbf{1} \supset \mathbf{2}]^{+\bullet}$ are stable. At intermediate potentials, i.e., in the range between -0.4 and $-0.6 \mathrm{~V}, \mathbf{2}^{+\bullet}$ and $[\mathbf{1} \supset \mathbf{2}]^{2+}$ are the thermodynamically stable species. Therefore, in agreement with the concept presented in Figure $1 \mathrm{~b}$, the autonomous threading and dethreading of $\mathbf{2}^{2+}$ and 1 can occur in the following scenario. The cathode is kept at $-0.8 \mathrm{~V}$, a potential at which [1 $\supset \mathbf{2}]^{2+}$ is reduced to [1 $\left.\supset \mathbf{2}\right]^{+\bullet}$. Owing to the fast dethreading of the latter and the diffusion time from cathode to anode (which we can control by changing the inter-electrode distance $d$ ), free $\mathbf{2}^{+\bullet}$ arrives at the anode, maintained at $0 \mathrm{~V}$, where it is promptly reoxidized to $\mathbf{2}^{2+}$. Eventually, rethreading of the latter into $\mathbf{1}$ regenerates the [1 $\left.\supset \mathbf{2}\right]^{2+}$ complex (which is stable near the anode) on a time scale faster than diffusion of free $\mathbf{2}^{2+}$ to the cathode. The pseudorotaxane can then reach the cathode by diffusion, thereby closing the autonomous threading-dethreading cycle.

a
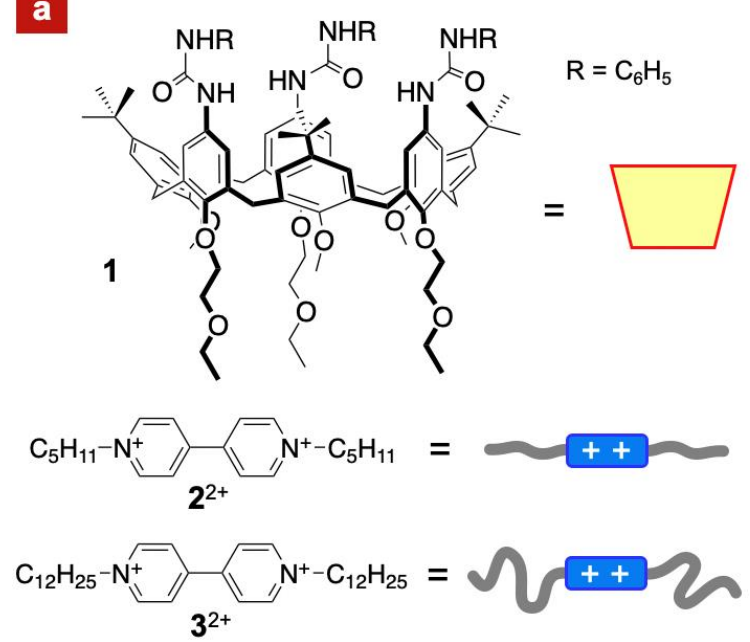

c

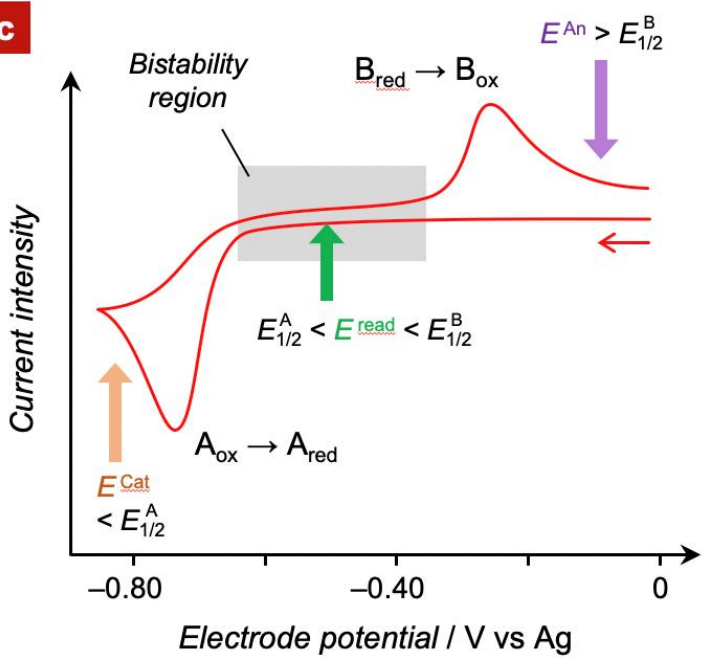

b
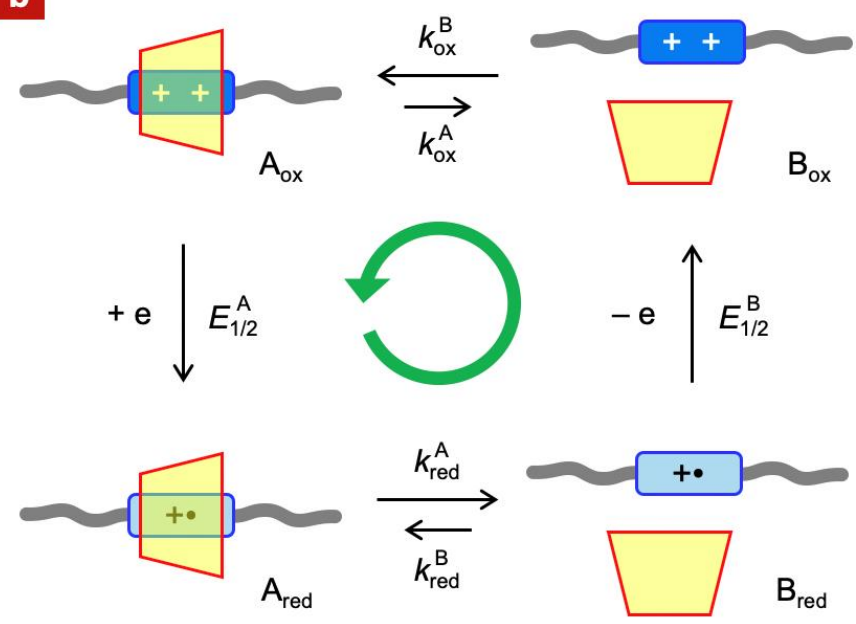

d

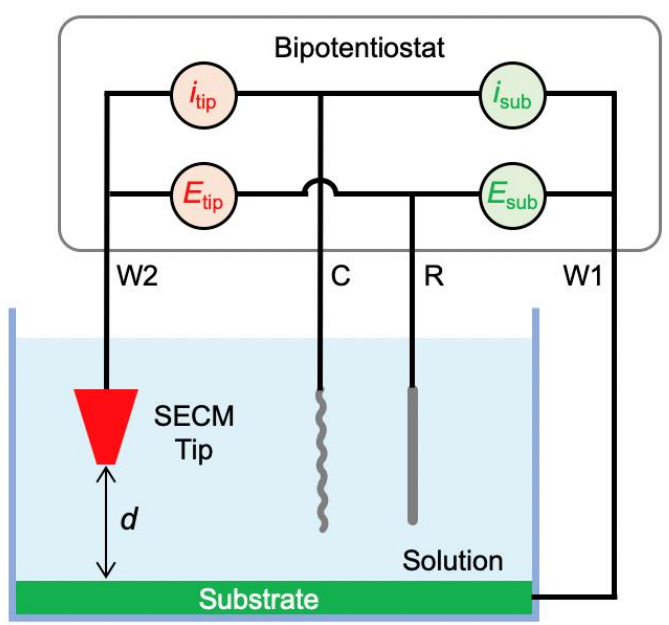

Figure 2. General properties and setup of the investigated system. (a) Chemical formulas of the molecular components; tosylate salts of $\mathbf{2}^{2+}$ and $\mathbf{3}^{2+}$ were employed. (b) Network of the self-assembly and redox reactions that describe the operation of the system. The stability constants of the pseudorotaxanes in the oxidized and reduced states are respectively $K_{\mathrm{ox}}=k^{\mathrm{B}}{ }_{\text {ox }} / k^{\mathrm{A}}$ ox and $K_{\text {red }}=k_{\text {red }}^{\mathrm{B}} / k^{\mathrm{A}}$ red, with $K_{\text {ox }}>K_{\text {red. }}$. (c) Relevant 
features of a typical cyclic voltammetric curve recorded on a solution of $\mathbf{1}$ and $\mathbf{2}^{2+}$. Electrode potential values, in $\mathrm{V}$ versus an $\mathrm{Ag}$ pseudo-reference electrode, are indicative; actual figures depend on experimental conditions such as solvent and scan rate. The labels are coherent with those employed in Figure 1b. (d) Scheme of the scanning electrochemical microscope (SECM) with bipotentiostatic configuration. The circuit allows the simultaneous and independent control of the potential of the two working electrodes (W1, substrate, and $\mathrm{W} 2$, tip). The distance between the substrate and the tip (d) can be easily varied by employing the SECM stepping motors and piezoelectrical components. $C$ and $R$ are respectively the counter and reference electrodes. The solution is contained in a deoxygenated and sealed electrochemical cell.

\section{Experimental design and setup}

Our SECM setup (Figure $2 \mathrm{~d}$ ) allows to control the potential of a substrate electrode $\left(E_{\text {sub }}\right)$ and that of the probe electrode $\left(E_{\text {tip }}\right)$ simultaneously, and vary them in a programmed fashion. We will refer to the probe electrode (a platinum UME disk) as the tip. For example, $E_{\text {sub }}$ can be set at a constant value, while scanning $E_{\text {tip }}$ to perform a CV using the tip as the working electrode, thus allowing the detection of the species that has formed at the substrate and has diffused to the tip. The distance between the tip and the substrate $(d)$ is controlled by measuring the tip current while approaching the substrate (probe approach curves, see the $\mathrm{SI}$ ). In our experimental setup this distance is set at $25 \mu \mathrm{m}$, unless otherwise stated.

Other important elements for the present discussion are indeed the diffusion rates, and the threading and dethreading rates. In particular, the autonomous mechanism described above requires that the dethreading of electrogenerated $[\mathbf{1} \supset \mathbf{2}]^{+\bullet}$ (i.e., $A_{\text {red }} \rightarrow B_{\text {red }}$ in Figure $1 b$ ) be faster than its diffusion to the anode. Similarly, the rethreading of $2^{2+}$ produced at the anode (i.e., $B_{o x} \rightarrow A_{o x}$ in Figure 1 b) should occur before free $\mathbf{2}^{2+}$ reaches the cathode. On the other hand, the species formed upon the redox process that occurs at one electrode must diffuse to the other electrode fast enough to be detected on the time scale of the electrochemical experiment. The values of the diffusion coefficient $(D)$ of $\mathbf{2}^{2+}$ and $[\mathbf{1} \supset \mathbf{2}]^{2+}$, determined by voltammetric measurements under the same conditions used in the SECM experiments (see the SI), are respectively $9.6 \times 10^{-6}$ and $6.5 \times 10^{-6}$ $\mathrm{cm}^{2} \mathrm{~s}^{-1}$, and are reasonably assumed to be equal to those of the corresponding reduced forms, $\mathbf{2}^{+\bullet}$ and $[\mathbf{1} \supset \mathbf{2}]^{+\bullet} \cdot{ }^{47}$ From the expression of the mean square displacement, one can thus estimate that an inter-electrode distance $d$ of $25 \mu \mathrm{m}$ is covered in about 3-400 ms. The threading rate constant, determined by stopped-flow absorption experiments in $\mathrm{CH}_{2} \mathrm{Cl}_{2}$ with a 100 -fold excess of TBAPF 6 , resulted to be $k^{B}{ }_{o x}=2.3 \times 10^{6} \mathrm{M}^{-1} \mathrm{~s}^{-1}$. This means that at the concentrations employed in the SECM experiments, the threading half-life of 1 and $2^{2+}$ is $3 \mathrm{~ms}$, much faster than the inter-electrode diffusion time. A similar conclusion holds for the dethreading of [1 $\mathbf{2}]^{+\bullet}$ (see the $\mathrm{SI}$ ).

These analyses show that (i) the redox reaction performed at one electrode is rapidly followed by a chemical transformation to restore equilibrium, and (ii) the species generated in the process can be promptly detected at the other electrode. Hence, the selected pseudorotaxane appears to fulfill the requirements for electrically-driven autonomous operation with our SECM setup.

\section{Demonstration of autonomous operation}

SECM is commonly used to probe with the UME the processes occurring at the substrate electrode. Yet, the fact that the species produced at one electrode reach the other electrode was also confirmed under the currently employed experimental conditions. To prove that the species produced at the substrate electrode reach the tip electrode, a solution of $\mathbf{2}^{2+}$ was initially analyzed. In particular, a CV was performed at the tip electrode, while the substrate was kept at a fixed 
potential of $-0.8 \mathrm{~V}$, a potential at which the stable species is $\mathbf{2}^{+\bullet}$ (Figure 3a). The CV scan performed at the tip reveals an oxidation process (positive current) coherent with the re-oxidation of $\mathbf{2}^{+\bullet}$ (Figure $3 a$, red curve), indicating that the species produced at the substrate electrode arrive at the tip under the employed conditions.
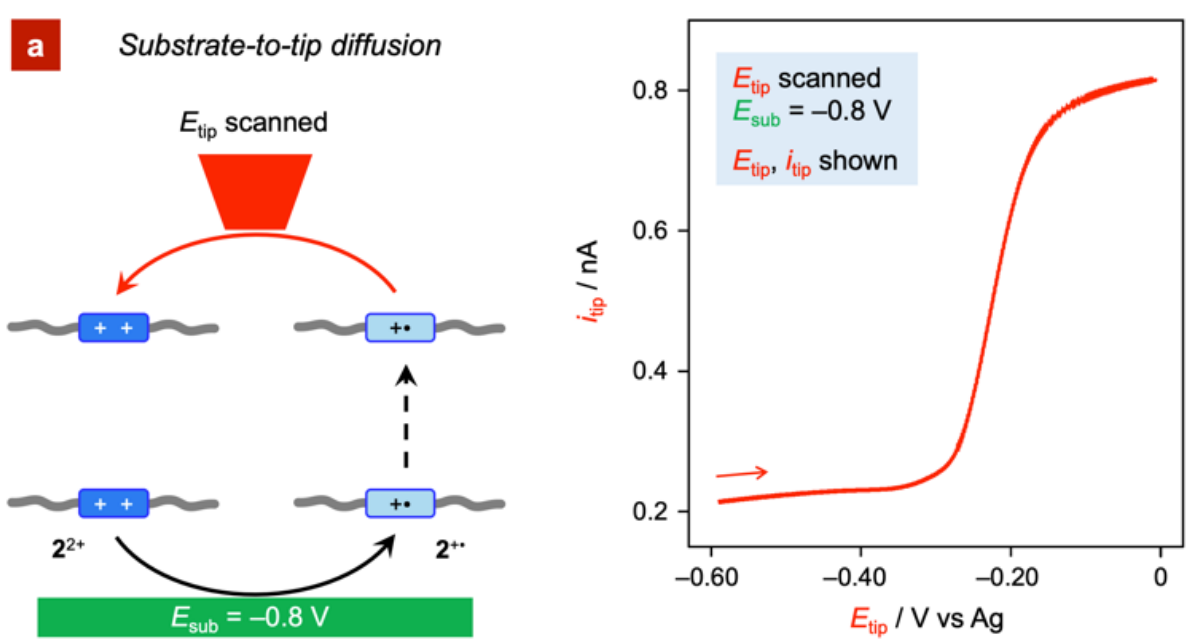

b Tip-to-substrate diffusion
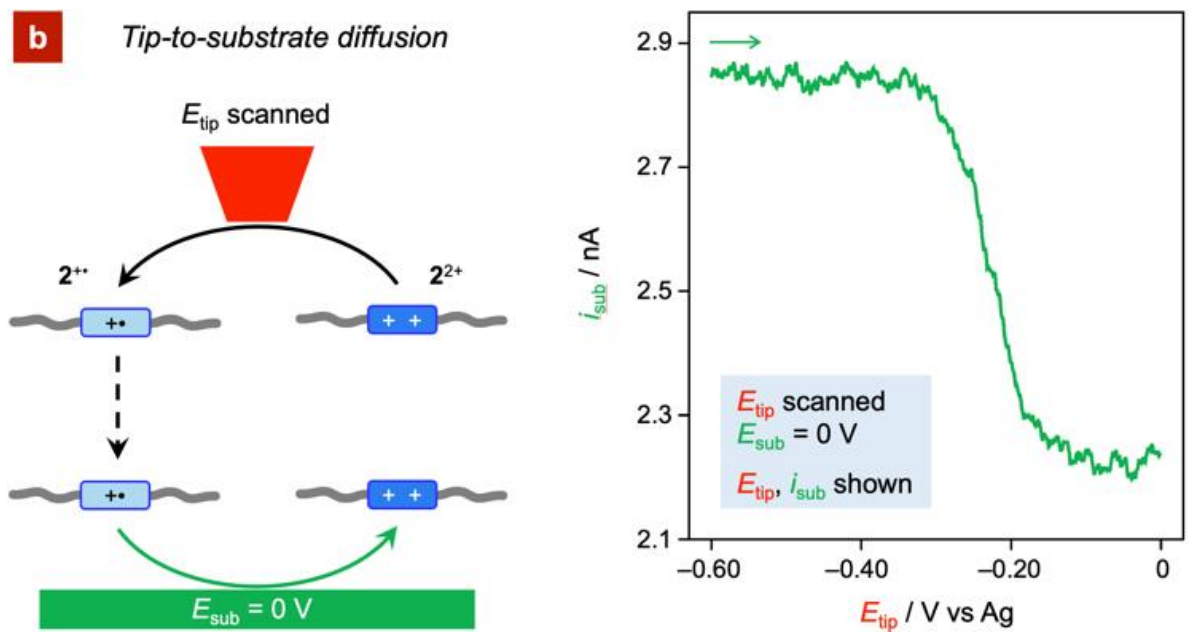

Figure 3. Electrogeneration of species at one electrode and detection at the other electrode. (a) A CV performed at the tip electrode detects the presence of $\mathbf{2}^{+\bullet}$ formed upon reduction of $\mathbf{2}^{2+}$ at $-0.8 \mathrm{~V}$ at the substrate electrode and diffused to the tip. (b) The positive current recorded at the substrate electrode reveals the oxidation of $\mathbf{2}^{+\bullet}$ produced during a CV scan at the tip. Note that oxidation and reduction currents have positive and negative sign, respectively. Inter-electrode diffusion is represented by dashed arrows, while the colored arrows indicate the electron-transfer processes monitored in the correspondingly colored voltammetric curve. Experimental conditions: $\mathrm{CH}_{2} \mathrm{Cl}_{2}$, r.t., $\mathbf{2}^{2+} 240 \mu \mathrm{M}, \mathrm{TBAPF}_{6} 24 \mathrm{mM}, 100 \mathrm{mV} \mathrm{s}{ }^{-1}, d=25 \mu \mathrm{m}$, potential values are referred to an $\mathrm{Ag}$ pseudo-reference electrode.

We also confirmed that the species produced at the tip electrode reach the substrate electrode. To this aim, the latter was set at $0 \mathrm{~V}$, and its current was monitored while the tip potential was scanned between 0 and $-0.6 \mathrm{~V}$, thus reducing $2^{2+}$ to $\mathbf{2}^{+\bullet}$ (Figure $3 \mathrm{~b}$ ). During the reduction scan at the tip, an oxidative current was recorded at the substrate, confirming that the species produced at the tip - in this case $\mathbf{2}^{+\bullet}$ - arrive at the substrate electrode, where re-oxidation to $\mathbf{2}^{2+}$ takes place (Figure 
$3 b$, green curve). Having established that the two electrodes are sufficiently near to allow the species produced at the electrode surface to diffuse from one electrode to the other, we proceeded demonstrating that the threading and dethreading processes occur faster than diffusion to the other electrode.

Specifically, regarding the dethreading process, it has to be demonstrated that following reduction of the pseudorotaxane $[\mathbf{1} \supset \mathbf{2}]^{2+}$, dethreading occurs before the reduced pseudototaxane reaches the tip. In this scenario, when the pseudorotaxane is reduced at the substrate electrode, the tip detects the free, reduced axle $\mathbf{2}^{+\bullet}$ and not the reduced pseudorotaxane. In order to prove this sequence of events, a solution of $1: 2^{2+} 1.6: 1$ was investigated. Under this condition, the majority of axle $\mathbf{2}^{2+}$ is present in the form of pseudorotaxane [1 $\left.\supset 2\right]^{2+}$. As shown in Figure $4 a$, the substrate was kept at $-0.8 \mathrm{~V}$, a potential at which the reduced pseudorotaxane [1 $\supset \mathbf{2}]^{+\bullet}$ is produced. Simultaneously, a CV was performed at the tip electrode, scanning between -0.6 and $0 \mathrm{~V}$. This CV scan is aimed at detecting the free axle $2^{+\bullet}$; an oxidative current was indeed recorded at $-0.3 \mathrm{~V}$ (Figure $4 \mathrm{a}$, red curve), i.e., a potential much closer to the one for oxidation of free $\mathbf{2}^{+\bullet}(-0.25 \mathrm{~V})$ rather than that of the parent pseudorotaxane $(-0.8 \mathrm{~V})$. This observation indicates that the reduction-induced dethreading of $\mathbf{2}^{+\bullet}$ occurs faster than diffusion to the other electrode.

To complete the requirements for autonomous operation, it has to be demonstrated that upon re-oxidation of $\mathbf{2}^{+\bullet}$ to $\mathbf{2}^{2+}$, threading is faster than diffusion to the opposite electrode. Gaining direct evidence of this process is far from trivial because, in principle, one should detect a newly formed pseudorotaxane $[\mathbf{1} \supset \mathbf{2}]^{2+}$ in a solution that contains a bulk amount of the same species. However, we reasoned that, in simple CV reduction experiments, during the return (anodic) scan, the uncomplexed guest $\mathbf{2}^{+\bullet}$ is detected, as evidenced by an oxidation wave occurring at a potential of ca. $-0.3 \mathrm{~V}$ (Figure $2 \mathrm{c}$ ). Therefore, when the potential of the substrate electrode is scanned, uncomplexed $\mathbf{2}^{2+}$ is generated during the return scan at potentials more positive than ca. $-0.3 \mathrm{~V}$. To assess whether the newly formed species reaches the tip electrode before or after forming the thermodynamically more stable pseudorotaxane $[\mathbf{1} \supset \mathbf{2}]^{2+}, E_{\text {tip }}$ was kept at $-0.6 \mathrm{~V}$, a value at which the free axle $\mathbf{2}^{2+}$ can be reduced, but not the $[\mathbf{1} \supset \mathbf{2}]^{2+}$ complex (Figure $4 \mathrm{~b}$ ). Indeed, no reduction current was observed under these conditions (Figure $4 \mathrm{~b}$, red trace), suggesting that rethreading occurs faster than diffusion of free $\mathbf{2}^{2+}$ towards the tip electrode.

Importantly, the same experiment was performed using axle $\mathbf{3}^{2+}$, i.e., an axle analog of $\mathbf{2}^{2+}$ bearing dodecyl alkyl chains instead of pentyl ones (Figure 1a), which impart a much slower threading rate with $1\left(k^{B}{ }_{o x}=2.6 \times 10^{2} \mathrm{M}^{-1} \mathrm{~s}^{-1}\right)$ compared with parent $2^{2+}\left(k^{\mathrm{B}}{ }_{\mathrm{ox}}=2.3 \times 10^{6} \mathrm{M}^{-1} \mathrm{~s}^{-1}\right) \cdot{ }^{45}$ In this case, a reduction current was detected at the tip potential (Figure $4 c$, red trace). This observation is coherent with $\mathbf{3}^{2+}$ reaching the tip electrode before rethreading, thus corroborating the above interpretation. This control experiment also highlights the effect of the selfassembly/disassembly dynamics on the electrochemical response of the system. To verify our analysis, we have performed numerical simulations using the experimentally determined values for redox processes, self-assembly reactions and diffusion coefficients. All the data presented in Figure 3 and 4 were reproduced, supporting our interpretation (see the SI). 
a Electrochemically induced dethreading
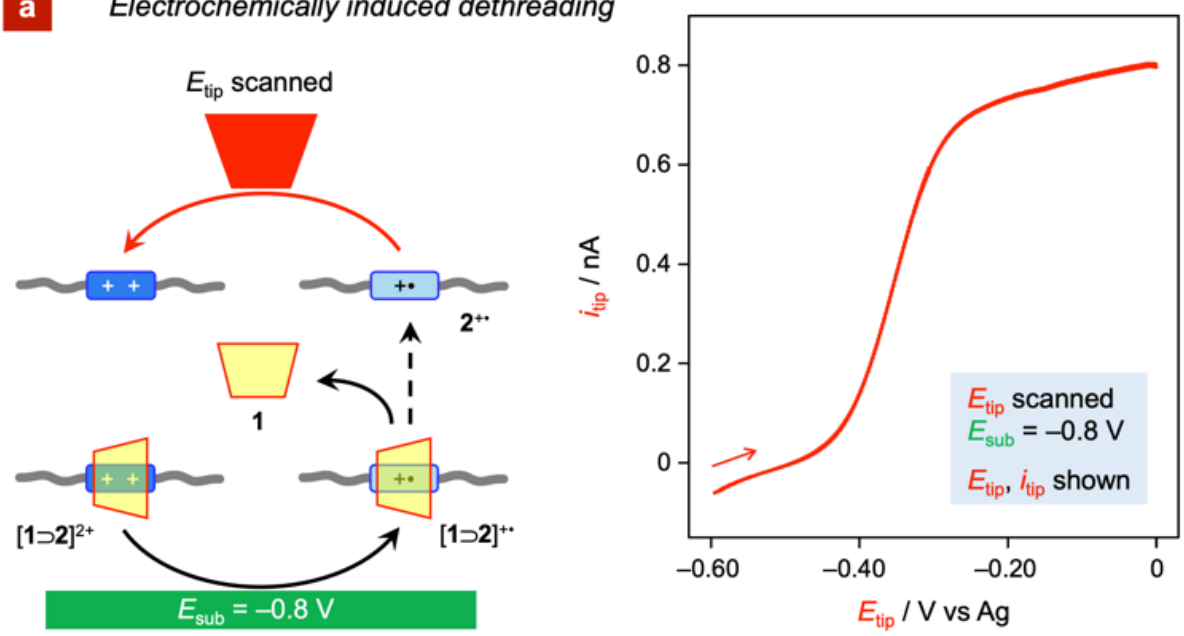

b Electrochemically induced threading
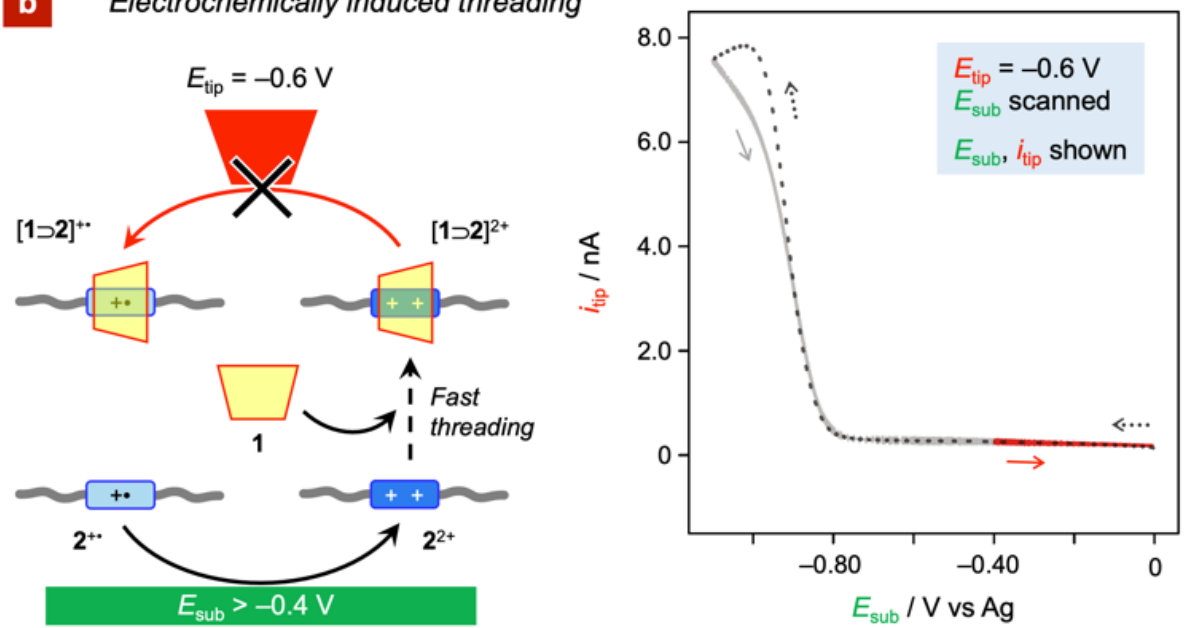

c Very slow threading - Control experiment
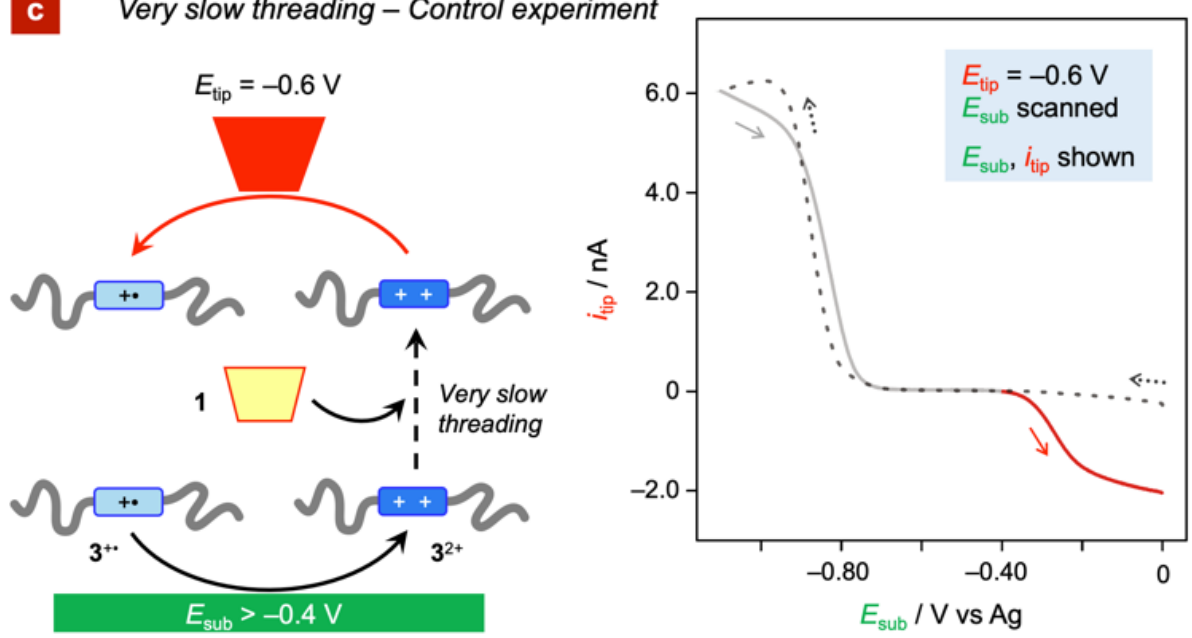

Figure 4. Demonstration of electrochemically induced threading-dethreading and subsequent interelectrode diffusion. (a) A CV performed at the tip electrode detects $\mathbf{2}^{+\cdot}$ formed at the substrate electrode upon reduction of $[\mathbf{1} \supset \mathbf{2}]^{2+}$ and subsequent dethreading. (b) Free $\mathbf{2}^{2+}$, generated from $\mathbf{2}^{+\bullet}$ during a CV scan at the substrate electrode, quickly rethreads with $\mathbf{1}$ on the diffusion time scale; the resulting $[\mathbf{1} \supset \mathbf{2}]^{2+}$ complex is not detected at the tip because it is electrochemically silent at $-0.6 \mathrm{~V}$. (c) The same experiment in (b) 
performed with guest $\mathbf{3}^{2+}$ in the place of $\mathbf{2}^{2+}$. The former is characterized by much slower threading kinetics than the latter; as a result, free $\mathbf{3}^{2+}$ produced at the substrate electrode does not undergo significant threading on the diffusion time scale, thus it is reduced at the tip. Note that oxidation and reduction currents have positive and negative sign, respectively. Inter-electrode diffusion is represented by dashed arrows, while the colored arrows indicate the electron-transfer processes monitored in the correspondingly colored voltammetric curve. For clarity, the forward (cathodic) scan in (b) and (c) is represented with a dotted line. Experimental conditions: $\mathrm{CH}_{2} \mathrm{Cl}_{2}$, r.t., $1380 \mu \mathrm{M}, \mathbf{2}^{2+}$ or $3^{2+} 240 \mu \mathrm{M}, \mathrm{TBAPF}_{6} 24 \mathrm{mM}, 100 \mathrm{mV} \mathrm{s}^{-1}, d=25$ (a) or $<4(\mathrm{~b}, \mathrm{c}) \mu \mathrm{m}$, potential values are referred to an Ag pseudo-reference electrode.

Overall, we have shown that the tip-substrate distance is sufficiently small to ensure the mutual communication of electroactive species, while large enough to ensure that threading and dethreading movements occur before reaching the opposite electrode by diffusion. Hence, an experiment can be performed to achieve the autonomous electrically driven operation of the molecular machine as depicted in Figure $1 \mathrm{~b}$. The substrate and tip electrodes were positioned at $d$ $=25 \mu \mathrm{m}$, their potentials were set respectively at -0.8 and $0 \mathrm{~V}$, and the tip current was monitored for a period of $250 \mathrm{~s}$ (Figure 5a). A quasi-stationary current of $0.85 \mathrm{nA}$ was measured, indicating that redox reactions occurred at the two electrodes.

a

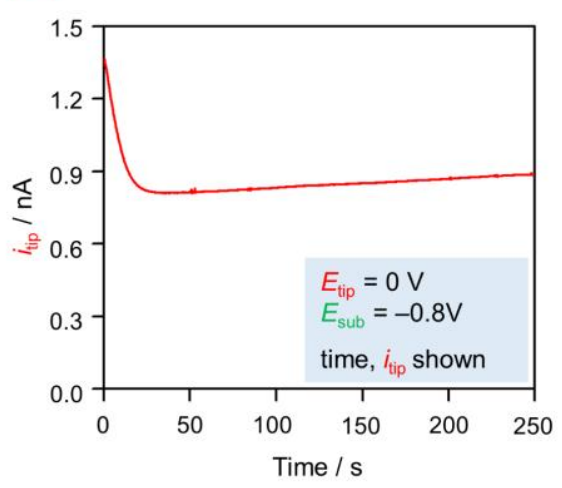

c

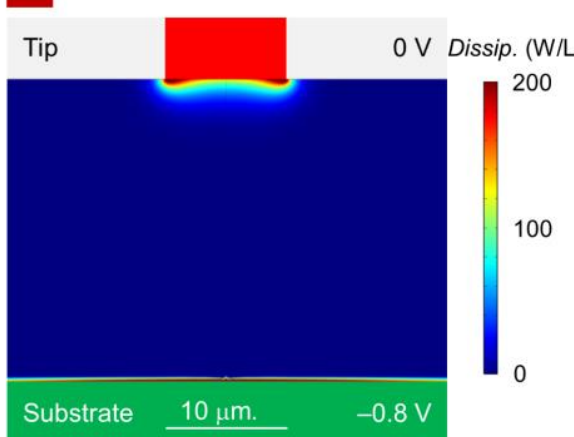

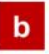
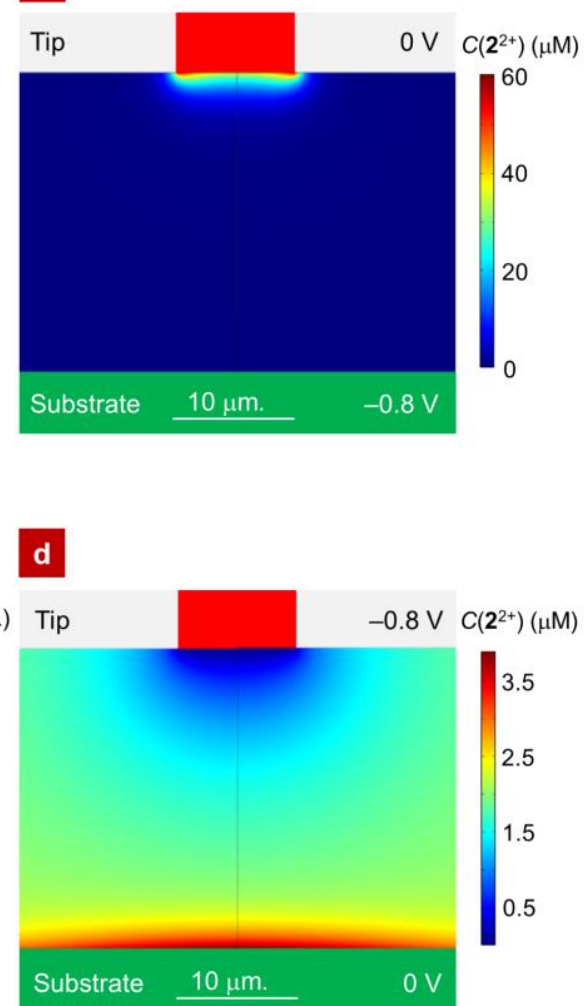

Figure 5. Autonomous electrochemical operation. (a) Current recorded at the tip electrode in an amperometric experiment performed by fixing tip and substrate potentials at 0 and $-0.8 \mathrm{~V}$, respectively. (b) Simulated concentration of $\mathbf{2}^{2+}$ at $\mathrm{t}=150 \mathrm{~s}$. (c) Simulated total dissipation (power per unit volume) associated with self-assembly reactions at $t=150 \mathrm{~s}$. (d) Simulated concentration of $\mathbf{2}^{2+}$ at $t=150 \mathrm{~s}$, with $E_{\text {tip }}=-0.8 \mathrm{~V}, E_{\text {sub }}$ $=0$ V. Experimental conditions: $\mathrm{CH}_{2} \mathrm{Cl}_{2}$, r.t., $1380 \mu \mathrm{M}, 2^{2+} 240 \mu \mathrm{M}, \mathrm{TBAPF}_{6} 24 \mathrm{mM}, 100 \mathrm{mV} \mathrm{s}^{-1}, d=25 \mu \mathrm{m}, E_{\text {tip }}$ $=0 \mathrm{~V}$ and $E_{\text {sub }}=-0.8 \mathrm{~V}(\mathrm{a}-\mathrm{c})$ or $E_{\text {tip }}=-0.8 \mathrm{~V}$ and $E_{\text {sub }}=0 \mathrm{~V}(\mathrm{~d})$, potential values are referred to an Ag pseudoreference electrode. 
Numeric simulations revealed the dynamic features of the observed non-equilibrium steadystate. Concentration maps indicate that $\mathbf{2}^{2+}$ is formed at the tip and readily depleted in its vicinity to form $[\mathbf{1} \supset \mathbf{2}]^{2+}$, which can diffuse unperturbed to the substrate (Figure $5 \mathrm{~b}$ and $\mathrm{SI}$ ). Analogously, $[\mathbf{1} \supset \mathbf{2}]^{+\bullet}$, is formed at the substrate and immediately disassembles. The net rates of self-assembly of $[\mathbf{1} \supset \mathbf{2}]^{2+}$ and $[\mathbf{1} \supset \mathbf{2}]^{+\bullet}$, i.e., chemical currents, quantitatively report on these processes. Their values change depending on the specific point in space that is considered, but are equal and opposite to $3.4 \mathrm{fmol} \mathrm{s}^{-1}$ when integrated over the entire volume comprised between the electrodes, i.e., the portion of space perturbed by the imposed non-equilibrium conditions (see the $\mathrm{SI}$ ). Comparison of this rate with the electric current reveals that, on average, 1.4 electrons transferred from the cathode to the anode cause one dethreading and one rethreading events. As a consequence, axles in proximity of the electrodes perform a threading-dethreading cycle every 26 seconds; this means that, on average, any given species can undergo about 10 threading-dethreading events on the experimentally observed timescale (250 s). The access to the dynamics of the systems allowed us to evaluate the efficiency at which the input energy might eventually be exploited, corresponding to the free energy dissipated by the machine as mechanical motion. ${ }^{48}$ This quantity corresponds to the energy dissipated in the self-assembly steps, which can be retrieved from the rates of assembly and disassembly and visualized (Figure $5 \mathrm{c}$ and $\mathrm{SI}$ ). Dissipation takes place in close proximity to both electrodes while the central part of the solution remains close to equilibrium. Comparison of the energy dissipated in the self-assembly steps with the electrical input energy, $W_{\text {in }}=n F \Delta E$ ( $n$, number of exchanged electrons; $F$, Faraday constant; $\Delta E$, electrode potential difference $=0.8 \mathrm{~V}$ ) affords an efficiency of 0.06. Remarkably, such energy efficiency is significantly higher than those of other autonomous artificial systems reported to date. . $^{3,4,5,6,49}$

We have operated our system also under opposite boundary conditions, i.e., $E_{\text {tip }}=-0.8$ and $E_{\text {sub }}$ $=0 \mathrm{~V}$, proving that multiple non-equilibrium steady-states are experimentally accessible using this system. ${ }^{50}$ While the recorded current $(-0.8 \mathrm{nA})$ is similar to the one measured under opposite boundary conditions, the distribution of species is completely different. This fact is evident when comparing the stationary concentrations of $\mathbf{2}^{2+}$ in the two cases (cf. Figure $5 b$ and $5 d$ ). Intriguingly, in this different operation mode the calculated efficiency is even higher, reaching 0.09. Since it is known that thermodynamic stabilities dictate the directionality in systems operating according to an energy ratchet mechanism, but not in those relying on information ratchet, we simulated the autonomous operation of an identical system in which the thermodynamic stabilities of oxidized and reduced pseudorotaxanes were inverted (see $\mathrm{SI}) .{ }^{21}$ As a result, inversion of the self-assembly current was also observed, validating the operation of this engine as an autonomous energy ratchet.

\section{Conclusion}

In summary, we have demonstrated the autonomous operation of an electrically-driven nanomachine, a pseudotoraxane performing threading and dethreading motions. Mechanical steps are part of a cyclic network of chemical reactions that occurs directionally under non-equilibrium conditions, operating as a chemical engine. ${ }^{51}$ As for other autonomous systems, the autonomous operation was established through a series of individually provable premises, taking advantage of a deductive logic mathematical proofs. ${ }^{4}$ The present system operates according to an energy ratchet mechanism, which requires alternating boundary conditions. Here, spatial asymmetry and diffusion provide such alternation. ${ }^{52}$ Therefore, system operation mode is fundamentally different from those of other autonomous systems. As a consequence, high engine operation efficiencies are obtained, up to 0.09 in the optimal operation setup. The strategy is entirely general, as it could be applied to any redox-driven nanomachine, including molecular pumps ${ }^{53}$ able to perform work 
repetitively. ${ }^{11,12}$ Even besides molecular machines, the same strategy can operate non-equilibrium supramolecular systems relying on electrical energy. ${ }^{38,54}$ Ultimately, unlocking the autonomous use of electrical energy by molecular machines brings nanoscience closer to modern-day technology.

Acknowledgements. The authors would like to thank Prof. Marwan Fahs and Dr. Sara Dal Cengio for support in using Comsol Multiphysics, Leonardo Andreoni and Chiara Taticchi for preliminary tests, and Emanuele Penocchio and Prof. R. Dean Astumian for fruitful discussions. This work was supported by the European Union's H2020 Research Programme (ERC AdG n. 692981), and the Interdisciplinary Thematic Institute ITI-CSC via the IdEx Unistra (ANR-10-IDEX-0002) within the program Investissement d'Avenir.

Keywords: molecular machines $\bullet$ non-equilibrium processes $\bullet \mathrm{SECM} \bullet$ electrochemistry $\bullet$ calixarene

\section{References}

1 Yewdall, N. A.; Mason, A. F; van Hest, J. C. M. The Hallmarks of Living Systems: Towards Creating Artificial Cells. Interface Focus 2018, 8, 20180023.

2 Grzybowski, B. A.; Huck, W. T. S. The Nanotechnology of Life-inspired Systems. Nat. Nanotechnol. 2016, 11, 585592.

3. Koumura, N.; Zijlstra, R. W. J.; van Delden, R. A.; Harada, N.; Feringa, B. L. Light-Driven Monodirectional Molecular Rotor. Nature 1999, 401, 152-155.

4. Wilson, M. R.; Sola, J.; Carlone, A.; Goldup, S. M.; Lebrasseur, N.; Leigh, D. A. An Autonomous Chemically Fueled Small-Molecule Motor. Nature 2016, 534, 235-240.

5. Ragazzon, G.; Baroncini, M.; Silvi, S.; Venturi, M.; Credi, A. Light-Powered Autonomous and Directional Molecular Motion of a Dissipative Self-Assembling System. Nat. Nanotechnol. 2015, 10, 70-75.

6. Amano, S.; Fielden, S. D. P.; Leigh, D. A. A Catalysis-Driven Artificial Molecular Pump. Nature 2021, 594, 529-534.

7. Tierney, H. L.; Murphy, C. J.; Jewell, A. D.; Baber, A. E.; Iski, E. V.; Khodaverdian, H. Y.; McGuire, A. F.; Klebanov, N.; Sykes, E. C. H. Experimental Demonstration of a Single-Molecule Electric Motor. Nat. Nanotechnol. 2011, 6, 625-629.

8. Perera, U. G. E.; Ample, F.; Kersell, H.; Zhang, Y.; Vives, G.; Echeverria, J.; Grisolia, M.; Rapenne, G.; Joachim, C.; Hla, S. W. Controlled Clockwise and Anticlockwise Rotational Switching of a Molecular Motor. Nat. Nanotechnol. 2013, $8,46-51$.

9 Amemiya, S.; Bard, A. J.; Fan, F. R. F.; Mirkin, M. V.; Unwin, P. R. Scanning Electrochemical Microscopy. Annu. Rev. Anal. Chem. 2008, 1, 95-131.

10 Polcari, D.; Dauphin-Ducharme, P.; Mauzeroll, J. Scanning Electrochemical Microscopy: A Comprehensive Review of Experimental Parameters from 1989 to 2015. Chem. Rev. 2016, 116, 22, 13234-13278.

11. Cheng, C.; McGonigal, P. R.; Schneebeli, S. T.; Li, H.; Vermeulen, N. A.; Ke, C.; Stoddart, J. F. An Artificial Molecular Pump. Nat. Nanotechnol. 2015, 10, 547-553.

12. Qiu, Y.; Song, B.; Pezzato, C.; Shen, D.; Liu, W.; Zhang, L.; Feng, Y.; Guo, Q. H.; Cai, K.; Li, W.; Chen, H.; Nguyen, M. T.; Shi, Y.; Cheng, C.; Astumian, R. D.; Li, X.; Stoddart, J. F. A Precise Polyrotaxane Synthesizer. Science 2020, 368, 1247-1253.

13. Astumian, R. D. Design Principles for Brownian Molecular Machines: How to Swim in Molasses and Walk in a Hurricane. Phys. Chem. Chem. Phys. 2007, 9, 5067-5083.

14. Erbas-Cakmak, S.; Leigh, D. A.; McTernan, C. T.; Nussbaumer, A. L. Artificial Molecular Machines. Chem. Rev. 2015, $115,10081-10206$. 
15. Kassem, S.; Van Leeuwen, T.; Lubbe, A. S.; Wilson, M. R.; Feringa, B. L.; Leigh, D. A. Artificial Molecular Motors. Chem. Soc. Rev. 2017, 46, 2592-2621.

16. Baroncini, M.; Casimiro, L.; de Vet, C.; Groppi, J.; Silvi, S.; Credi, A. Making and Operating Molecular Machines: A Multidisciplinary Challenge. ChemistryOpen 2018, 7, 169-179.

17. Brown, A. I.; Sivak, D. A. Theory of Nonequilibrium Free Energy Transduction by Molecular Machines. Chem. Rev. 2020, 120, 434-459.

18. Andreoni, L.; Baroncini, M.; Groppi, J.; Silvi, S.; Taticchi, C.; Credi, A. Photochemical Energy Conversion with Artificial Molecular Machines. Energy Fuels 2021, doi: 10.1021/acs.energyfuels.1c02921.

19. Rao, R.; Esposito, M. Nonequilibrium Thermodynamics of Chemical Reaction Networks: Wisdom from Stochastic Thermodynamics. Phys. Rev. X 2016, 6, 041064.

20. Ragazzon, G.; Prins, L. J. Energy Consumption in Chemical Fuel-Driven Self-Assembly. Nat. Nanotechnol. 2018, 13, 882-889.

21. Astumian, R. D. Kinetic Asymmetry Allows Macromolecular Catalysts to Drive an Information Ratchet. Nat. Commun. 2019, 10, 3837.

22. Astumian, R. D.; Mukherjee, S.; Warshel, A. The Physics and Physical Chemistry of Molecular Machines. ChemPhysChem 2016, 1719-1741.

23. Astumian, R. D. Running on Information. Nat. Nanotechnol. 2016, 11, 582-583.

24. Greb, L.; Lehn, J.-M. Light-Driven Molecular Motors: Imines as Four-Step or Two-Step Unidirectional Rotors. J. Am. Chem. Soc. 2014, 136, 13114-13117.

25. Petermayer, C.; Dube, H. Indigoid Photoswitches: Visible Light Responsive Molecular Tools. Acc. Chem. Res. 2018, $51,1153-1163$.

26. Chen, Y.; Wang, M.; Mao, C. An Autonomous DNA Nanomotor Powered by a DNA Enzyme. Angew. Chem. Int. Ed. 2004, 43, 3554-3557.

27. Balzani, V.; Clemente-León, M.; Credi, A.; Ferrer, B.; Venturi, M.; Flood, A. H.; Stoddart, J. F. Autonomous Artificial Nanomotor Powered by Sunlight. Proc. Natl. Acad. Sci. U. S. A. 2006, 103, 1178-1183.

28 Serreli, V.; Lee, C.; Kay, E. R.; Leigh, D. A. A molecular information ratchet. Nature 2007, 445, 523-527.

29. Biagini, C.; Capocasa, G.; Cataldi, V.; Del Giudice, D.; Mandolini, L.; Di Stefano, S. The Hydrolysis of the Anhydride of 2-Cyano-2-phenylpropanoic Acid Triggers the Repeated Back and Forth Motions of an Acid-Base Operated Molecular Switch. Chem. Eur. J. 2019, 25, 15205-15211.

30 Borsley, S.; Leigh, D. A.; Roberts, B. M. W. A Doubly Kinetically-Gated Information Ratchet Autonomously Driven by Carbodiimide Hydration. J. Am. Chem. Soc. 2021, 143, 11, 4414-4420.

31 Green, S. J.; Bath, J.; Turberfield, A. J. Coordinated Chemomechanical Cycles: A Mechanism for Autonomous Molecular Motion. Phys. Rev. Lett. 2008, 101, 238101.

32. Li, Q.; Fuks, G.; Moulin, E.; Maaloum, M.; Rawiso, M.; Kulic, I.; Foy, J. T.; Giuseppone, N. Macroscopic Contraction of a Gel Induced by the Integrated Motion of Light-Driven Molecular Motors. Nat. Nanotechnol. 2015, 10, 161165.

33. Ikegami, T.; Kageyama, Y.; Obara, K.; Takeda, S. Dissipative and Autonomous Square-Wave Self-Oscillation of a Macroscopic Hybrid Self-Assembly under Continuous Light Irradiation. Angew. Chem. Int. Ed. 2016, 55, 8239-8243.

34. Nummelin, S.; Shen, B.; Piskunen, P.; Liu, Q.; Kostiainen, M. A.; Linko, V. Robotic DNA Nanostructures. ACS Synth. Biol. 2020, 9, 1923-1940.

35 Zhang, L.; Marcos, V.; Leigh, D. A. Molecular Machines with Bio-inspired Mechanisms. Proc. Nat. Acad. Sci. USA 2018 115, 9397-9404.

36 Aprahamian, I. The Future of Molecular Machines. ACS Cent. Sci. 2020, 6, 347-358.

37 Baroncini, M.; Silvi, S.; Credi, A. Photo- and Redox-Driven Artificial Molecular Motors. Chem. Rev. 2020, 120, 200268.

38. Yoshida, R.; Takahashi, T.; Yamaguchi, T.; Ichijo, H. Self-Oscillating Gel. J. Am. Chem. Soc. 1996, 118, 5134-5135. 
39. Yoshida, R.; Ueki, T. Evolution of self-oscillating polymer gels as autonomous polymer systems. NPG Asia Mater. 2014, 6, e107.

40. J. Leira-Iglesias, A. Tassoni, T. Adachi, M. Stich, T. M. Hermans, Oscillations, Travelling Fronts and Patterns in a Supramolecular System. Nat. Nanotechnol. 2018, 13, 1021-1028.

41. Ogden, W. A.; Guan, Z. Redox Chemical-Fueled Dissipative Self-Assembly of Active Materials. ChemSystemsChem 2020, 2, e1900030.

42. Krabbenborg, S. O.; Veerbeek, J.; Huskens, J. Spatially Controlled Out-of-Equilibrium Host-Guest System under Electrochemical Control. Chem. Eur. J. 2015, 21, 9638-9644.

43 Amatore, C. Electrochemistry at Ultramicroelectrodes in Physical Electrochemistry; Rubinstein, I., Ed.; Marcel Dekker, New York, 1995, Chapter 4.

44. Wittstock, G.; Burchardt, M.; Pust, S. E.; Shen, Y.; Zhao, C. Scanning Electrochemical Microscopy for Direct Imaging of Reaction Rates. Angew. Chem. Int. Ed. 2007, 46, 1584-1617.

45. Arduini, A.; Bussolati, R.; Credi, A.; Secchi, A.; Silvi, S.; Semeraro, M.; Venturi, M. Toward Directionally Controlled Molecular Motions and Kinetic Intra- and Intermolecular Self-Sorting: Threading Processes of Nonsymmetric Wheel and Axle Components. J. Am. Chem. Soc. 2013, 135, 9924-9930.

46. Credi, A.; Dumas, S.; Silvi, S.; Venturi, M.; Arduini, A.; Pochini, A.; Secchi, A. Viologen-Calix[6]arene Pseudorotaxanes. Ion-Pair Recognition and Threading/Dethreading Molecular Motions. J. Org. Chem. 2004, 69, 5881-5887.

47. Cook, S. K.; Horrocks, B. R. Heterogeneous Electron-Transfer Rates for the Reduction of Viologen Derivatives at Platinum and Bismuth Electrodes in Acetonitrile. ChemElectroChem 2017, 4, 320-331.

48. Amano, S.; Esposito, M.; Kreidt, E.; Leigh, D. A.; Penocchio, E.; Roberts, B. M. W. Insights from an Information Thermodynamics Analysis of a Synthetic Molecular Motor. ChemRxiv 2021, DOI: 10.33774/chemrxiv-2021-60k1r.

49. Larson, A. M.; Balema, T. A.; Zahl, P.; Schilling, A. C.; Stacchiola, D. J.; Sykes, C. H. Hypothetical Efficiency of Electrical to Mechanical Energy Transfer during Individual Stochastic Molecular Switching Events. ACS Nano 2020, 14, 16558-16564.

50. Sorrenti, A.; Leira-Iglesias, J.; Sato, A.; Hermans, T. M. Non-Equilibrium Steady States in Supramolecular Polymerization. Nat. Commun. 2017, 8, 15899.

51. Amano, S.; Borsley, S.; Leigh, D. A.; Sun, Z. Chemical engines: Driving Systems away from Equilibrium through Catalyst Reaction Cycles. Nat. Nanotechnol. 2021, accepted.

52. Chen, R.; Neri, S.; Prins, L. J. Enhanced Catalytic Activity Under Non-Equilibrium Conditions. Nat. Nanotechnol. 2020, $15,868-874$.

53. Feng, Y.; Ovalle, M.; Seale, J. S. W.; Lee, C. K.; Kim, D. J.; Astumian, R. D.; Stoddart, J. F. Molecular Pumps and Motors. J. Am. Chem. Soc. 2021, 143, 5569-5591.

54. Selmani, S.; Schwartz, E.; Mulvey, J. T.; Wei, H.; Grosvirt-dramen, A.; Gibson, W.; Hochbaum, A. I.; Patterson, J. P.; Ragan, R.; Guan, Z. Electrically Fueled Active Supramolecular Materials. ChemrXiv 2021, DOI: 10.33774/chemrxiv2021-h6ls0. 